\title{
Performance and cost of a Renal Cancer Specialist Multidisciplinary Team Meeting: Results from 1500 discussions
}

Authors: Joana B Neves ${ }^{*}, 1,2$, Scott Shepherd ${ }^{*}$, , David Cullen ${ }^{4}$, Tom Powles ${ }^{5,6}$, Michael Aitchison ${ }^{7,8}$, Maxine G B Tran ${ }^{7,9}$, On behalf of the Specialist Centre for Kidney Cancer, Royal Free London NHS Foundation Trust

*Both authors contributed equally to this study

\section{Affiliations:}

${ }^{1}$ Clinical research fellow in Renal Cancer, Specialist Centre for Kidney Cancer, Royal Free London NHS Foundation Trust, London, UK

${ }^{2}$ Research associate in Urology, Division of Surgery and Interventional Science, University College London

${ }^{3}$ Specialty registrar in medical oncology, The Royal Marsden NHS Foundation Trust, London, UK; former clinical fellow in oncology, Royal Free London NHS Foundation Trust, London, UK

${ }^{4}$ Clinical Specialist Nurse Lead in Renal Cancer, Specialist Centre for Kidney Cancer, Royal Free London NHS Foundation Trust, London, UK

${ }^{5}$ Consultant oncologist, Barts NHS Health and The Royal Free London NHS Foundation Trust, London, UK

${ }^{6}$ Clinical Professor of Genitourinary Oncology, Barts Cancer Institute, London, UK ${ }^{7}$ Consultant urologist, Specialist Centre for Kidney Cancer, Royal Free London NHS Foundation Trust, London, UK

${ }^{8}$ Director, Specialist Centre for Kidney Cancer, Royal Free London NHS Foundation Trust, London, UK 
${ }^{9}$ Senior lecturer in Renal Cancer, Division of Surgery and Interventional Science, University College London, London, UK

\section{Corresponding author:}

Maxine GB Tran, PhD, FRCS(Urol)

Address:

Royal Free Hospital

Pond street, $9^{\text {th }}$ floor,

Division of Surgery and Interventional Science, UCL,

London

NW3 2QG

United Kingdom

Email: m.tran@ucl.ac.uk

Conflict of interest: TP has received funding from AstraZeneca, Merck Sharp and Dohme, Pfizer, Roche, Novartis, and Bristol-Myers Squibb, and honoraria from AstraZeneca, Merck Sharp and Dohme, Pfizer, Exelixis, Lilly, and Janssen. The remaining authors declare no conflicts of interest.

Financial or other support: This research received no specific grant from any funding agency in the public, commercial, or not-for-profit sectors.

Ethics: Research Ethics Committee approval is not required for reporting clinical audits. 
Informed consent: Not sought as only anonymised data was used for this analysis.

Contributions and garantorship: MT is responsible for the study design and is guarantor of the study. JBN and SS acquired the data. JBN, and SS analysed the data. DC, TP, MA and MT provided expertise on renal cancer and on the renal cancer multidisciplinary team process. All authors contributed to the critical discussion of results and writing of the manuscript.

Acknowledgments: JBN received funding from St Peters' Trust for Kidney, Bladder and Prostate Research (Royal Free Charity) and MGBT from the Nick Maude Memorial Fund/Kidney Cancer UK and Facing Up 2 Kidney Cancer charities.

\section{List of where the study was previously presented:}

- Poster presented at Public Health England National Cancer Registration and Analysis Service Cancer Data and Outcomes Conference 2017, UK

- Eposter moderated presentation at Société Internationale d'Urologie (SIU) meeting 2017

- Poster moderated presentation at American Urological Association (AUA) meeting 2018 


\section{Abstract}

Objectives: To report on the performance and cost of a surgeon-led renal cancer specialist multidisciplinary team meeting at a high-volume centre.

Materials and methods: Retrospective analysis of 1500 consecutive cases discussed from 02/09/2015 onwards. Performance was assessed as the number of cases where a clinical recommendation was made. The cost per meeting, discussion, and patient were calculated using the mid-point of pay band attributable to the attendees (NHS pay scales 2015).

Results: 1500 discussions occurred over 34 meetings (933 patients: $61.7 \%$ male; mean age 63.8). Above a quarter of discussions ( $n=399,26.6 \%)$ were new referrals. Each patient's case was discussed a mean of 1.6 times, the majority being discussed once $(n=563,60.3 \%)$. In 93.3\% discussions a clinical recommendation was made. Only 100 discussions (6.7\%) were deferred due to incomplete clinical information. $11.1 \%(n=166)$ of cases were discharged. The average cost was: $£ 141,901$ per year, $£ 2,729$ per meeting, £62 per case discussed, and £99 per patient.

Conclusion: One discussion was usually sufficient to decide management; deferral was uncommon; and, given the low discharge rate, referrals seemed appropriate. The cost per patient was modest and represented good value in providing a focused and shared clinical decision-making pathway for renal cancer patients.

Level of evidence: $2 \mathrm{C}$

\section{Keywords (MeSH)}

Kidney neoplasms, patient care team, patient care management, costs, cost analysis 


\section{Introduction}

Multidisciplinary team (MDT) meetings are structured assemblies of individuals from different disciplines that occur at scheduled times with the aim of discussing the clinical management of a given patient ${ }^{1}$. These meetings (also known as tumour boards in other countries such as the USA) were established as a means to improve patient outcomes and develop a critical mass of knowledge by transferring the diagnostic and management decision from one individual to a group of experts from complementary disciplines. Based on this concept they were adopted as standard of care and, since the early 2000's, it is mandated in the UK that all patients diagnosed with cancer are reviewed at MDT meetings ${ }^{1}$. MDT meetings must be regularly audited in the UK and have been shown to change patient management ${ }^{3}$, increase patient satisfaction $^{4}$, and may contribute towards better clinical outcomes ${ }^{5}$. Furthermore, a recent discrete choice experiment involving patients, health professionals and the public found that access to a specialist MDT was a particular preference when selecting a specialist cancer surgery service provider ${ }^{6}$.

There is evidence that centralisation of cancer surgical services is occurring on a global scale, including Europe and the USA. In the UK, particularly in London and Manchester, prostate, bladder, kidney and oesophageal cancer care have been rearranged into centralised high-volume centres where excellent levels of clinical and organisational expertise are expected to further improve treatment outcomes whilst also reducing costs 6,7 .

At a time when the health services, including the National Health Service (NHS), are under unprecedented budget pressure, the ability to combine rationalisation of costs with high quality care and clinical benefit appears attractive. 
The aim of our work is to use standardised metrics and report on the performance and cost efficiency of the surgeon-led specialist MDT (sMDT) meeting for the North Central and North East London renal cancer centre. 


\section{Materials and methods}

\section{The renal cancer specialist MDT meeting}

The renal cancer specialist MDT (sMDT) meeting is organised according to NHS recommendations. It is a weekly three-hour meeting hosted by The Royal Free Hospital (Royal Free London NHS Foundation Trust). All patients with confirmed or a high suspicion index of upper tract urological cancer from 13 referring hospital trusts across North Central and North East London are reviewed at this meeting. After the meeting, an outcome report is issued per case discussed and disseminated to referring clinicians. Discussion at the meeting precedes the clinic appointment with the patient and thus any informed discussion with the patient regarding the ensuing management options available usually occurs after MDT discussion.

Participants of the meeting include both core and extended members from various medical fields (urology, oncology, radiology, interventional radiology, histopathology and nephrology). Core members are attendees that sit throughout the duration of the meeting and whose presence is necessary to establish quorum. These include the meeting chair (a urology consultant), consultant urologists, consultant radiologists, consultant oncologists, clinical nurse specialists, and SMDT meeting coordinator. Extended members are attendees that sit on the meeting either for a limited period of time to participate in the discussion of their referred cases or throughout the duration of the meeting but whose presence does not count towards establishing a minimum quorum necessary for decision making. Some extended members attend the meeting solely via videoconference. Additionally, each referring centre provides administrative support via a local MDT coordinator to organise the list of cases to be discussed and ensure all relevant clinical information (history, 
examination, scan images, pathology reports and/or slides) is available at the time of the meeting.

\section{The NHS pay scale}

In the NHS, base annual salaries for all staff are predefined nationwide according to a scale ${ }^{8}$. This scale is further subdivided into bands and each band into spines. A range of bands is attributed to each type of job. Previous salary and level of expertise define the exact band each employee is allocated to, for example, a junior doctor is paid according to band 7 , while consultants are paid band 8 to 9 . The number of years working on the job defines the spine payment within each band (first year on the job corresponds to spine 1 , second to spine 2, and so forth).

\section{Data collection and analysis}

We retrospectively reviewed 1500 consecutive case discussions at the renal cancer sMDT meeting starting from 02/09/2015, and conducted descriptive analysis of patient demographics, referring hospital, referring specialty, reason for discussion, average number of discussions per meeting, number of discussions per patient in the time period considered, number of cases deferred to subsequent meetings, and number of cases discharged back to the community care. Cases were categorised according to previous referral to the meeting into new referrals (no previous discussion prior to 02/09/2015) and previously discussed cases (previous discussion prior to 02/09/2015).

As previously described ${ }^{9}$, performance of the meeting was assessed by calculating the rate of cases where a clinical recommendation was made (for example, requirement of further imaging scans, treatment/management recommendation, 
discharge, or post-treatment follow up scheme). Meeting decision implementation was not assessed. As MDT discussion usually precedes the clinic appointment with the patient, information on suitable management options made by the referring physician was in the main not available. Thus, it was not possible to assess if the sMDT ratified the referring physician's management decision.

To calculate the cost of running the meeting three factors were considered: the member attendance records of relevant meetings; the time spent at the meeting by core and extended members ( $3 \mathrm{~h}$ for core members and the average time spent by each extended member at the last three meetings included in the analysis); and the time spent preparing or actioning on outcomes from the meeting. These are the costing metrics advised by the NHS to calculate MDT meeting $\operatorname{cost}^{10}$. MDT coordinators and lead core members (urology, oncology, radiology, pathology, nursing) provided information regarding the average amount of time per week devoted to preparing case discussions and actioning on meeting outcomes. All lead core members and 4 out of 13 MDT coordinators responded to the request for information. When an estimate of preparation time was not provided, this was estimated as a function of the number of cases per referring hospital discussed per meeting. Each member and MDT coordinator were classified according to banding using the NHS pay scales from 1 April $2015^{8}$. The mid-spine value of each band was taken into consideration to calculate cost. When there were two mid-spine values, the lowest one was considered. London allowance was added to this value.

The cost of each meeting was calculated as a function the NHS salary pay per hour of each coordinator and member per hour based on the three factors named above. The cost per case discussed, and per patient was calculated. The annual cost of the sMDT meeting was extrapolated. 
The specialist renal cancer service at Royal Free Hospital was established in 2014, after the UK mandate that all patients diagnosed with cancer must be discussed at a MDT, thus no control group of cases not discussed at the sMDT was available for outcome comparison.

Reporting was done in accordance with the Revised Standards for Quality Improvement Reporting Excellence (SQUIRE 2.0) ${ }^{11}$.

Funding: none

Ethics: Research Ethics Committee approval is not required for reporting clinical audits. 


\section{Results}

One thousand and five hundred case discussions took place over 34 meetings $(02 / 09 / 2015$ to $20 / 04 / 2016)$. All meetings had quorum. Overall, $1375(91.7 \%)$ discussions were done in the context of disease localised to the kidney and $125(8.3 \%)$ were held in the context of metastatic disease. These discussions represented a cohort of 933 patients with a mean age of 63.8 (interquartile range (IQR) 24; minimum 14; maximum 96) and where $61.7 \%$ were male $(n=576)$. Royal Free London NHS Foundation Trust referred most patients to the sMDT meeting $(n=538,57.7 \%)$, followed by University College London Hospitals Trust $(n=108,11.6 \%$; Figure 1). Urology was the specialty that referred the majority of patients ( $n=720,77.2 \%)$, followed by renal medicine $(n=52,5.6 \%)$, and oncology $(n=45,4.8 \%$; Figure 2$)$.

On average, 44 case discussions took place per meeting, with an average of 4 minutes spent per case discussion. Just above a quarter of discussions ( $n=399$, $26.6 \%$ ) were new referrals. While most discussions were based on reviewing imaging scans, more than one in four $(n=416,27.7 \%)$ represented discussions to decide management based on biopsy or surgical pathology results.

Each patient's case was discussed a mean of 1.6 times: the majority of patients required one $(n=563,60.3 \%)$ or two case discussions $(n=234,25.1 \%)$ during the period studied. Eighty-nine (9.5\%) patients required three discussions, thirty-four $(3.6 \%)$ required 4 discussions, nine (1\%) required 5 , one $(0.1 \%)$ required 6 , and three $(0.3 \%)$ required 7 discussions.

Regarding meeting outcomes, in nearly two thirds of discussions ( $n=980$, $65.3 \%$ ) an intervention recommendation was made (such as renal tumour biopsy, active surveillance, surgery, cryotherapy and/or systemic therapy). The post-operative 
follow-up scheme was decided in 155 discussions (10.3\%). Overall, 11.1\% ( $n=166)$ of cases were discharged from care in the period considered. Ninety-nine (6.6\%) cases required further imaging prior to management recommendation. Only 100 discussions $(6.7 \%)$ were deferred due to incomplete clinical data, such as unavailability of imaging scans or pathology reports or slides for review. Thus, $93.3 \%$ of sMDT discussions resulted in clinical recommendations, which was the chosen performance outcome measure for this study.

The renal cancer sMDT meeting has 15 core members and 17 extended members (Table 1), with annual salaries varying from NHS Clinical Salary Band 4 to 9 plus London Allowance. Four out of 13 MDT coordinators reported the time taken to prepare and action on outcomes from the meeting (range from 0.5 to $20 \mathrm{~h}$ a week). For the remaining coordinators, time was estimated as a function of the number of cases per referring hospital discussed per meeting. Preparing and actioning on meeting outcomes involved $8.5 \mathrm{~h}$ of consultant time (urology $2.5 \mathrm{~h}$, oncology $0.5 \mathrm{~h}$, histopathology $1.5 \mathrm{~h}$, radiology $4 \mathrm{~h}$ ) and $5 \mathrm{~h}$ of clinical nurse specialist time. Based on the number of hours spent preparing for the meeting, the attendance record of meetings, and the amount of time each member spent at the meeting, the estimated average cost of the meeting was: $£ 141,901$ per year, £2,729 per meeting, £62 per case discussed, and £99 per patient. 


\section{Discussion}

Our analysis shows that our surgeon-lead renal cancer SMDT meeting is efficient and provides good value. In 1500 discussions over 34 meetings and spanning over 8 months, a clinical recommendation was made in $93.3 \%$ of discussions. Generally, one or two discussions were usually sufficient to make management recommendations, deferral of cases was uncommon, and, given the low discharge rate, referrals to the meeting seemed appropriate. Additionally, the meeting expenditure seemed reasonable: while the headline annual cost for the sMDT meeting seems high $£ 141,901$ ), the cost per patient is modest (£99) and is less than the cost of an outpatient clinic appointment within the $\mathrm{NHS}^{12}$.

Cases that required only one sMDT discussion likely represent situations where patients who had been discussed at the meeting before 02/09/2015 needed review regarding follow up requirements (such as deciding a surveillance plan after surgery, or to review new images for patients on surveillance) or where recommendations were made that did not require repeat SMDT discussion (initiation of systemic treatment, watchful waiting, or best supportive care, or discharge). Several reasons could explain the need for three or more discussions in $14.6 \%$ of patients. The wide time span of the study (over 8 months) likely includes re-review of patients with clinical progression on surveillance schemes and allows coverage of the small renal mass clinical pathway adopted by the specialist centre; whereby patients very frequently start their management by having a renal tumour biopsy, the results of which are reviewed at the MDT (second discussion), that can be followed by active treatment, such as surgery, that generates a third SMDT discussion to ascertain the best post-operative follow up scheme based on surgical histology. The minority of patients discussed four 
or more times likely represent highly complex situations, where multiple additional investigations where required and/or complications from treatment arose.

The average time of 4 minutes per case discussion most likely reflects a wide time range that correlates positively with case complexity. Notwithstanding, this illustrates the fast pace of the meeting and the importance of pre-meeting case preparation. It may also illustrate that the clinical decision-making process for some of the cases was very straightforward, questioning the utility of discussing all cancer cases at the MDT. However, even for what seems the most straightforward scenario for a patient diagnosed with a renal mass, the management decision process can become more difficult than initially apparent. Consideration of lesion location and size may limit the technical feasibility of certain types of intervention such as renal tumour biopsy, partial nephrectomy, or ablative treatment. Likewise, present and previous medical history can dictate the choice of active surveillance over intervention, preclude the start of systemic treatment, or expose the need for further clinical investigations or treatments prior to decision of management of the renal lesion. One could advocate that given that $91.7 \%$ discussions were done in the context of disease localised to the kidney that the clinical decision making relies mostly on the surgeons and radiologists present at the meeting and that a separate meeting including pathologists and oncologists would suffice to discuss histology results (27.7\% of discussions) and metastatic disease settings ( $8.3 \%$ of discussions). While the idea of streamlining MDTs is under discussion at present ${ }^{13}$, and is something we are considering at our centre, it is still unclear how this should be implemented. Often, a case is reclassified from "straightforward" to "complex" precisely because there was a MDT discussion where all key members were involved and contributed towards this decision. If a pathway outside MDT discussion were to be implemented for straightforward 
situations, it is unclear who and how these would be triaged and what criteria should warrant MDT discussion. Likewise, given that MDT discussion is now considered the gold standard in the UK, it is not known if patients would accept to have their care diverge from that pathway. Further studies involving all stakeholders, including patients, are required to fully critically appraise the MDT discussion pathway and develop streamlining tools.

There are two main limitations to our cost calculations: one is that an estimate of the gross salary per member was used to calculate cost (as opposed to using the actual salary of each member); the second is that only costs in salaries were taken into account and other costs, such as videoconferencing and other overheads, were not considered. These factors may have contributed to a lower meeting-associated expenditure when compared to previous UK reports ${ }^{14}$. Nonetheless, considering the similar member composition of sMDT meetings in the UK across different cancer types and the use of standardised NHS metrics for MDT cost calculation ${ }^{10}$, the overall salary associated cost calculated for this meeting may be used for reference indication for the cost of other specialist clinical service meetings.

Given the current UK practice to discuss all patients at cancer MDT meetings, within the NHS it is not possible to formally compare the clinical efficacy and cost effectiveness of these meetings to the previous single clinician decision pathway using a contemporaneous comparative cohort. Historical cohort studies have many limitations. Additionally, as our specialist centre and its MDT were established after the UK mandate, the use of an historical cohort was not possible. On one hand, studies suggest that MDT meetings contribute to faster and more appropriate patient management ${ }^{3-5}$, particularly in unusual or rare situations ${ }^{15}$, are cost beneficial ${ }^{16}$, and lead to increased patient satisfation ${ }^{4}$. On the other hand, poor study design, 
publication bias, and heterogeneity between study outcomes may prevent a factual assessment of the benefits (or lack thereof) of MDT discussions ${ }^{17}$. Additionally, some have pointed out that the need to discuss all diagnostic and treatment options for straightforward cases limits the resources available to discuss difficult situations ${ }^{18}$ and that the absence of the patient at the MDT decision-making threatens autonomy ${ }^{19}$. In fact, the UK MDT mandate was established before strong evidence of effectiveness was established, limiting accurate evaluation of its clinical benefit ${ }^{20}$. Our study is unable to address these controversies. However, we do show that, at a modest cost, the renal cancer SMDT meeting addresses the clinical management of a large volume of patients, has a lower deferral rate than previous reports ${ }^{14}$, and in more than $90 \%$ of cases defines focused and clinically valid management options that can be presented and discussed with patients. This data is encouraging and supports the continued use of a multidisciplinary decision-making platform for all renal cancer patients at a highvolume expert centre. Streamlining MDT discussions is under consideration ${ }^{13}$ but further discussions are needed between all stakeholders to understand how this can be implemented without compromising patient care. 


\section{References}

1. The manual for cancer services. Department of Health, London, 2004.

2. Improving outcomes in urological cancer - NICE. https://www.nice.org.uk/guidance/csg2/resources/improving-outcomes-inurological-cancers-pdf-773372413 [accessed September 2018].

3. Kurpad R, Kim W, Rathmell WK, et al. A multidisciplinary approach to the management of urologic malignancies: does it influence diagnostic and treatment decisions? Urologic oncology $2011 ; 29: 378-82$.

4. El Turabi A, Abel GA, Roland $M$, et al. Variation in reported experience of involvement in cancer treatment decision making: evidence from the National Cancer Patient Experience Survey. Br J Cancer 2013; 109(3):780-7.

5. Kesson EM, Allardice GM, George WD, et al. Effects of multidisciplinary team working on breast cancer survival: retrospective, comparative, interventional cohort study of 13722 women. BMJ 2012;344:e2718.

6. Vallejo-Torres L, Melnychuk M, Vindrola-Padros C, Aitchison M, Clarke CS, Fulop NJ, Hines J, Levermore C, Maddineni SB, Perry C, Pritchard-Jones K, Ramsay AIG, Shackley DC, Morris S. Discrete-choice experiment to analyse preferences for centralizing specialist cancer surgery services. Br J Surg 2018;105(5):587-96

7. London Cancer Specialist Services Reconfiguration: A case for change in specialist cancer $\quad$ services. $\quad$ https://www.england.nhs.uk/wpcontent/uploads/2014/03/cancer-clin-props.pdf [accessed April 2017].

8. NHS terms and conditions of service handbook - Amendment number 35: Pay and conditions circulars number 1/2015, number 2/2015 and number 3/2015. 
http://www.nhsemployers.org/ /media/Employers/Documents/Pay

and reward/AfC_tc_of_service_handbook_fb.pdf [accessed April 2017].

9. Lamb BW, Green JS, Benn J, et al. Improving decision making in multidisciplinary tumor boards: prospective longitudinal evaluation of a multicomponent intervention for 1,421 patients. J Am Coll Surg 2013;217(3):412-20.

10. Healthcare costing standards for England. https://improvement.nhs.uk/documents/2377/Healthcare_costing_standards__acute_CMs2.pdf [accessed September 2018].

11. Ogrinc G, Davies L, Goodman D, Batalden P, Davidoff F, Stevens D. SQUIRE 2.0 (Standards for QUality Improvement Reporting Excellence): revised publication guidelines from a detailed consensus process. BMJ Qual Saf 2016;25:986-92.

12. NHS reference costs 2015 to 2016. https://www.gov.uk/government/publications/nhs-reference-costs-2015-to-2016 [accessed April 2017].

13. Achieving World-Class Cancer Outcomes: Taking the strategy forward. https://www.england.nhs.uk/wp-content/uploads/2016/05/cancer-strategy.pdf [accessed September 2018].

14. De leso PB, Coward JI, Letsa I, et al. A study of the decision outcomes and financial costs of multidisciplinary team meetings (MDMs) in oncology. British Journal of Cancer 2013;109:2295-300.

15. Sooriakumaran P, Dick JA, Thompson AC, et al. The Central Urology Multidisciplinary Team - Is it Time to Change the Referral Criteria? An Audit of Practice in a District General Hospital in London. Annals of The Royal College of Surgeons of England 2009;91:700-2. 
16. Ke KM, Blazeby JM, Strong S, et al. Are multidisciplinary teams in secondary care cost-effective? A systematic review of the literature. Cost effectiveness and resource allocation 2013;11:7.

17. Taplin SH, Weaver S, Salas E, et al. Reviewing cancer care team effectiveness. Journal of Oncology Practice 2015;11:239-46.

18. Munro AJ. Multidisciplinary Team Meetings in Cancer Care: An Idea Whose Time has Gone?. Clin Oncol 2015:27:728-31.

19. Coulter A, Collins A. Making shared decision-making a reality - No decision about me, without me. The King's Fund, London, 2011.

20. Fleissig A, Jenkins V, Catt S, Fallowfield L. Multidisciplinary teams in cancer care: are they effective in the UK? Lancet Oncol 2006;7(11):935-43.

\section{Figure and table legends}

Figure 1 - Referring trusts

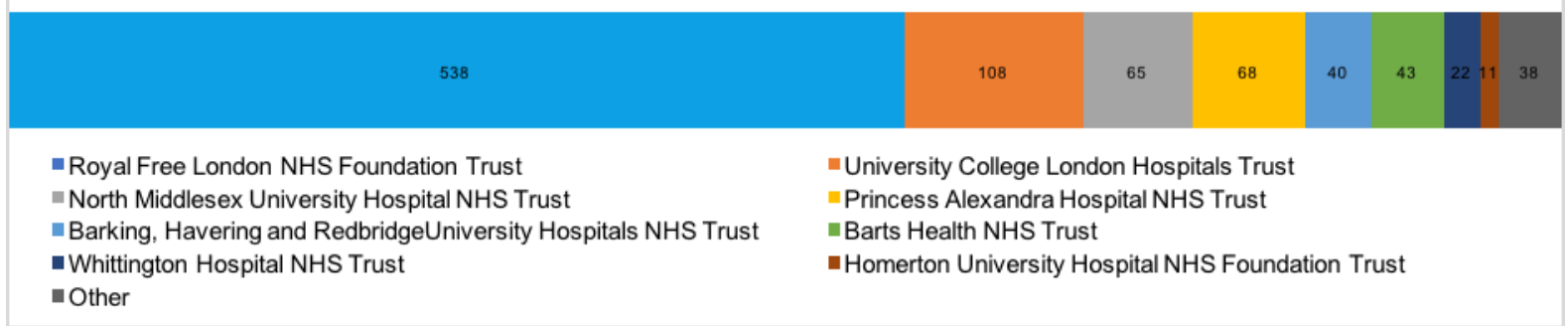

Figure 2 - Referring specialties

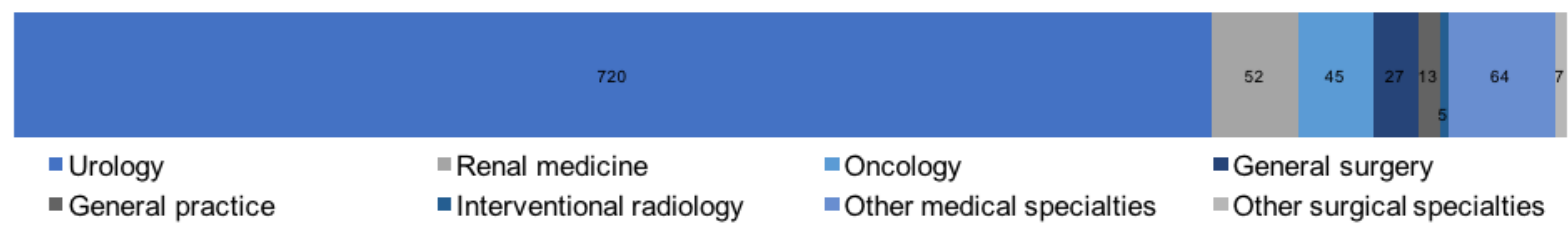




\begin{tabular}{|l|l|}
\hline Core Members & Extended Members \\
\hline MDT Coordinator & Urology consultant x3 \\
\hline MDT Chair (Urology consultant) & Radiology consultant \\
\hline Oncology Lead (Oncology consultant) & Nephrology consultant \\
\hline Histopathology Lead (Histopathology & Histopathology consultant x3 \\
\hline Imaging Lead (Radiology consultant) & Interventional radiology consultant \\
\hline Urology consultant x3 & Specialty registrar in Urology \\
\hline Oncology consultant x2 & Specialty registrar in Oncology \\
\hline Radiology consultant & Specialty registrar in Histopathology \\
\hline Interventional radiology consultant & Clinical nurse specialist in urology \\
\hline Clinical nurse specialist in renal cancer & Clinical nurse specialist in oncology \\
\hline Clinical nurse specialist in renal cancer & Clinical research nurse \\
\hline Clinical nurse specialist in oncology & Clinical trial practitioner \\
\hline & Renal Cancer navigator \\
\hline
\end{tabular}

Table 1 - Renal cancer specialist MDT meeting members 